\title{
Learning Algorithms for Separable Approximations of Discrete Stochastic Optimization Problems
}

\author{
Warren Powell \\ Department of Operations Research and Financial Engineering, Princeton University, \\ Princeton, New Jersey 08544, powell@ princeton.edu \\ Andrzej Ruszczyński \\ Department of Management Science and Information Systems, Rutgers University, \\ 94 Rockefeller Road, Piscataway, New Jersey 08854, rusz@ business.rutgers.edu \\ Huseyin Topaloglu \\ Department of Operations Research and Industrial Engineering, Cornell University, \\ Ithaca, New York 14853, topaloglu@orie.cornell.edu
}

\begin{abstract}
We propose the use of sequences of separable, piecewise linear approximations for solving nondifferentiable stochastic optimization problems. The approximations are constructed adaptively using a combination of stochastic subgradient information and possibly sample information on the objective function itself. We prove the convergence of several versions of such methods when the objective function is separable and has integer break points, and we illustrate their behavior on numerical examples. We then demonstrate the performance on nonseparable problems that arise in the context of two-stage stochastic programming problems, and demonstrate that these techniques provide near-optimal solutions with a very fast rate of convergence compared with other solution techniques.
\end{abstract}

Key words: stochastic programming; dynamic programming

MSC2000 subject classification: Primary: 90C15

OR/MS subject classification: Primary: Programming, stochastic

History: Received August 5, 2002; revised May 6, 2003, and November 28, 2003.

1. Introduction. We consider the following stochastic programming problem:

$$
\max _{x \in X} \mathbb{E} f(x, \omega),
$$

where $f: \mathbb{R}^{n} \times \Omega \rightarrow \mathbb{R},(\Omega, \mathscr{F}, \mathbb{P})$ is a probability space, and $\mathbb{E}$ denotes the expected value. We assume that the function $f$ is concave with respect to the first argument, and such that

$$
F(x)=\mathbb{E} f(x, \omega)
$$

is finite for every $x \in X$. We assume that for almost all $\omega$, all $i=1, \ldots, m$, and for all feasible integer values of $x_{j}$ for $j \neq i$, the function $f\left(x_{1}, \ldots, x_{i-1}, \cdot, x_{i+1}, \ldots, x_{n}, \omega\right)$ is piecewise linear with integer break points. We also assume that the set $X$ is closed, and that

$$
X \subseteq\left\{x \in \mathbb{R}^{n}: 0 \leq x_{i} \leq M_{i}, i=1, \ldots, n\right\} .
$$

Problems of this type arise in a variety of resource allocation problems. In the car distribution problem of railroads, for example, planners often face the problem of having to reposition empty cars before a customer order has been realized. Truckload carriers have to assign drivers to loads before knowing the opportunities available to the driver at the destination. The air mobility command needs logic for their simulators that will reposition aircraft back to locations where they might be needed, before actual demands are known.

All of these problems can be modeled as multistage stochastic linear programming problems. Experimental research (see, for example, Godfrey and Powell 2001) has demonstrated that the recourse function for these problems can be well approximated by sequences of piecewise linear, separable functions. These scalar functions can be approximated by techniques that use sample gradients, where the challenge is to maintain the concavity of the 
approximations. As a result of the need to use Monte Carlo samples, it is necessary to introduce steps that maintain the concavity after every update. This paper explores a class of such techniques and establishes conditions under which these approximations converge to the true function.

An important case is what is referred to as the two-stage stochastic program:

$$
\begin{aligned}
\max \langle c, x\rangle+\mathbb{E} Q(x, \omega) \\
\text { subject to } \quad A x=b, \\
x \geq 0,
\end{aligned}
$$

where

$$
\begin{gathered}
\qquad(x, \omega)=\max \langle q, y\rangle \\
\text { subject to } \quad \begin{aligned}
W y & =h(\omega)-T x, \\
y & \geq 0 .
\end{aligned}
\end{gathered}
$$

A special case of (2) is the two-stage stochastic program with network recourse, where the second-stage problem is the min-cost network flow problem. If $h$ takes integer values a.s., then this problem is a special case of (1). Another special case occurs when $f$ in (1) is a separable function of the form

$$
f(x, \omega)=\sum_{i=1}^{n} f_{i}\left(x_{i}, \omega\right) .
$$

This is the form most often taken in the context of classical resource allocation problems (Righter 1989) that involve the allocation of resources to independent activities subject to a common budget constraint.

To obtain the optimal solution to (1), one can consider building sequential approximations of $F$, say $F^{k}$. If the sequence $\left\{F^{k}\right\}$ converges to $F$ in an appropriate sense, then we can claim to have a procedure to solve (1). Alternatively, we may solve optimization problems of the form $x^{k} \in \arg \max _{x \in X} F^{k}(x)$, constructed in such a way that the sequence $\left\{x^{k}\right\}$ converges to $x^{*} \in \arg \max _{x \in X} F(x)$. In this case the sequence of functions $\left\{F^{k}\right\}$ does not necessarily converge to $F$, but $\left\{x^{k}\right\}$ may converge to an optimal (or nearly optimal) point. Of critical importance in practical applications is also the speed of convergence, a question that we treat on an experimental basis.

For our class of applications it is relatively easy, for a given $x^{k}$, to sample an elementary event $\omega^{k}$ and to calculate $f_{i}\left(x_{i}^{k}, \omega^{k}\right)$. Moreover, it is also easy to obtain information about the slope of $f_{i}\left(\cdot, \omega^{k}\right)$ at $x_{i}^{k}$ :

$$
v_{i}^{k}=f_{i}\left(x_{i}^{k}, \omega^{k}\right)-f_{i}\left(x_{i}^{k}-1, \omega^{k}\right) .
$$

It is difficult, however, to obtain the exact values of $\bar{f}_{i}(x)=\mathbb{E}\left\{f_{i}(x, \omega)\right\}$, because it involves the calculation of the expected value.

Example 1. Let $x_{i}$ denote the amount of resource allocated to activity $i$, where $i=$ $1, \ldots, n$. These amounts have to be chosen from the set

$$
X=\left\{x \in \mathbb{R}^{n}: x_{i} \in\left\{0,1, \ldots, M_{i}\right\}, i=1, \ldots, n, \sum_{i=1}^{n} x_{i} \leq b\right\} .
$$

For each activity $i$ there is a nonnegative integer random variable $D_{i}$ representing the demand. The reward associated with activity $i$ is defined as in the newsvendor problem:

$$
f_{i}\left(x_{i}, D_{i}\right)=q_{i} \min \left(x_{i}, D_{i}\right)-c_{i} x_{i},
$$


where $q_{i}>c_{i}>0$. Our objective is to allocate the resources in such a way that the expected reward, $F(x)=\mathbb{E} \sum_{i=1}^{n} f_{i}\left(x_{i}, D_{i}\right)$, is maximized, subject to the constraint $x \in X$. Porteus (1990) provides a thorough review of the newsvendor problem in the context of stochastic inventory models. The optimal solution of a single newsvendor problem can be expressed analytically, but this requires knowing the distribution of demand. An extensive literature has evolved to solve what is known as the censored newsvendor problem (where you only see the amount sold, not the actual demand). This literature (see, for example, Ding et al. 2002) requires assuming a parametric form for the demand distribution. The algorithm provided in this paper does not require any information about the demand distribution or a set of demand observations from which the demand distribution can empirically be constructed.

In such a problem we may sample a demand realization $D^{k}=\left(D_{1}^{k}, \ldots, D_{n}^{k}\right)$ and calculate

$$
v_{i}^{k}=\left\{\begin{array}{ll}
q_{i}-c_{i} & \text { if } x_{i}^{k} \leq D_{i}^{k}, \\
-c_{i} & \text { if } x_{i}^{k}>D_{i}^{k} .
\end{array} \quad i=1, \ldots, n .\right.
$$

We can also calculate the right slope estimate

$$
v_{i+}^{k}=\left\{\begin{array}{ll}
q_{i}-c_{i} & \text { if } x_{i}^{k}<D_{i}^{k}, \\
-c_{i} & \text { if } x_{i}^{k} \geq D_{i}^{k},
\end{array} \quad i=1, \ldots, n .\right.
$$

The information our algorithm uses is $\left\{x^{k}\right\},\left\{v^{k}\right\}$, and $\left\{v_{+}^{k}\right\}$, where $x^{k}, v^{k}$, and $v_{+}^{k}$, respectively, denote the vectors $\left(x_{1}^{k}, \ldots, x_{n}^{k}\right),\left(v_{1}^{k}, \ldots, v_{n}^{k}\right)$, and $\left(v_{1+}^{k}, \ldots, v_{n+}^{k}\right)$. To compute any $v_{i}^{k}$ or $v_{i+}^{k}$, we only need to know if $x_{i}^{k} \leq D_{i}^{k}$. For the newsvendor problem, this translates into knowing whether or not the newsvendor has sold all the newspapers rather than observing the exact value of the demand random variable. This is exactly the same situation addressed in the censored newsvendor problem. Therefore, the algorithm presented in this paper provides an asymptotically optimal solution to the censored newsvendor problem without requiring any particular form for the demand distribution.

Similar estimates can be generated in a slightly more complicated case, with the reward associated with activity $i$ defined as

$$
f_{i}\left(x_{i}, D_{i}\right)=q_{i}\left(\min \left(x_{i}, D_{i}\right)\right)-c_{i}\left(x_{i}\right),
$$

where $q_{i}(\cdot)$ is a concave piecewise linear function, and $c_{i}(\cdot)$ is a convex piecewise linear function, both with break points at $0,1, \ldots, M_{i}$.

There exists a wealth of numerical methods for stochastic programming problems. The first group are scenario methods, in which a sufficiently rich sample $\omega^{1}, \ldots, \omega^{N}$ is drawn from the space $\Omega$, and the expectation is approximated by the sample average:

$$
F^{N}(x)=\frac{1}{N} \sum_{\nu=1}^{N} f\left(x, \omega^{\nu}\right) .
$$

A discussion of these approaches can be found in Shapiro and Homem-De-Mello (2000), Korf and Wets (2001), and Kleywegt et al. (2002).

The second group of methods are stochastic subgradient methods, which use the fact that the random vector $v^{k}$ in (4) satisfies the relation

$$
\mathbb{E}\left\{v^{k} \mid x^{k}\right\} \in \partial F\left(x^{k}\right),
$$

where $\partial F$ is the subdifferential of $F$ (understood as the negative of the subdifferential of the convex function $-F$ ). Stochastic subgradient algorithms depend on updates of the form

$$
x^{k+1}=x^{k}+\alpha_{k} v^{k} .
$$


These methods make very many small updates to the current approximation by using the stochastic subgradients as directions, achieving convergence almost surely to the optimal point. A treatment of these methods can be found in Ermoliev (1988). Constraints can be treated by projection, by feasible direction techniques (Ruszczyński 1980), or by recursive linearization (Ruszczyński 1987).

Finally, Benders decomposition can be used to solve two-stage stochastic linear programs by approximating the recourse function $\mathbb{E} Q(x, \omega)$ with a series of cutting planes (Van Slyke and Wets 1969, Ruszczyński 1986, Higle and Sen 1991, Ruszczyński 2003, Chen and Powell 1999).

Our problem class is motivated by applications that require integer solutions. The introduction of uncertainty often has the effect of destroying the natural integer structure of many problems. All of the three classes of techniques mentioned above destroy the natural integrality either by how the recourse function is approximated (scenario methods and Benders decomposition) or the nature of the algorithm itself (stochastic subgradient methods). The method proposed in this paper handles integrality requirements very easily (Laporte and Louveaux 1993 show how integrality can be incorporated into Benders decomposition).

We propose to solve these problems by adaptively estimating, using sample subgradient information, and sequences of separable approximations that are piecewise linear, concave, and that have integer break points. We use the information gathered in iterations $1, \ldots, k$ to construct models $f_{i}^{k}(\cdot)$ of the expected value functions $\bar{f}_{i}(\cdot)=\mathbb{E}\left\{f_{i}(\cdot, \omega)\right\}, i=1, \ldots, n$. The next approximation to the solution is given by

$$
x^{k} \in \underset{x \in X}{\arg \max } \sum_{i=1}^{n} f_{i}^{k}\left(x_{i}\right) .
$$

An associated learning step provides information employed to update the models $f_{i}^{k}$. Such an approach is already known to be optimal if the objective function is continuously differentiable (Culioli and Cohen 1990, Cheung and Powell 2000), but there is no comparable result for nondifferentiable problems. While the relation of stochastic approximation type methods and learning is well known (see, e.g. Kushner and Yin 1997), the use of the structure (separability and concavity) allows here for the construction of particularly efficient methods.

Our solution strategy extends a line of research in stochastic resource allocation using separable approximations. This problem class has been most widely studied using the framework of two-stage stochastic programs with network recourse (Wallace 1986, 1987; Birge and Wallace 1988). Independently, separable, piecewise linear approximations have been proposed for discrete resource allocation problems that arise in the context of fleet management (Powell 1986, 1987, 1988). Frantzeskakis and Powell 1990 suggest a static, piecewise linear separable approximation for specially structured tree problems, a result that is generalized in Powell and Cheung (1994) and applied to multistage resource allocation problems in Cheung and Powell (1996). These methods, however, were not adaptive, which limited the quality of the solution. Powell and Carvalho (1998) provided an adaptive learning algorithm based on linear approximations, which was extended in Godfrey and Powell (2001) to an adaptive, piecewise linear approximation based on the CAVE algorithm. The CAVE algorithm provided exceptionally good experimental performance, but offered no provable results. Wallace (1987) introduces a piecewise linear upper bound for networks, a result that is generalized in Birge and Wallace (1988) for stochastic programs.

In this paper, we introduce and formally study the use of sequences of piecewise linear, separable approximations as a strategy for solving nondifferentiable stochastic optimization problems. As a byproduct, we produce a fast algorithm for problems such as two-stage stochastic programs with network recourse, a topic that was first studied in depth by Wallace (1986). We establish several important convergence results for the special case of separable objective functions, and show experimentally that the algorithm provides near-optimal, 
and often optimal, solutions for problems when the objective function is not separable, as would be the case with two-stage stochastic programs. Furthermore, the observed speed of convergence is much faster than techniques such as Benders decomposition, especially for higher dimensional problems.

This paper is divided into two parts. Sections $2-6$ deal exclusively with problems where the original objective function $F(x, \omega)$ is separable. While this problem class enjoys its own sets of applications (for example, in a variety of budget allocation problems), our interest in this special problem class arises primarily because we are able to prove some important convergence results. Section 2 presents the basic algorithm for learning piecewise linear, concave approximations (while maintaining concavity after every update), and proves convergence to the real function assuming that all points are sampled infinitely often. Section 3 provides a variation of the algorithm that combines gradient information with sample information on the function itself. In practical applications, we cannot generally guarantee that we will sample all points infinitely often, and this is not necessary to find the optimal solution. Section 4 proves convergence when we only sample the points $x^{k}$ generated by Equation (6). Section 5 shows how a certain projection required to maintain concavity can be implemented, and $\$ 6$ provides the results of a series of experiments that investigate the rate of convergence of variations of the algorithm.

The second part of the paper, given in $\$ 7$, focuses on nonseparable problems that arise in the context of two-stage stochastic programs. We cannot guarantee that our algorithm will produce the optimal solution for two-stage problems, but we show that the right separable approximation can produce the optimal solution, and we use this to develop a bound on our result. Numerical comparisons with Benders decomposition (which is optimal for noninteger versions of these problems) indicate that our approach may provide much faster convergence and optimal or very-near-optimal results.

2. Learning concave functions of one variable. We start from the description and analysis of the basic learning algorithm for a concave piecewise linear function of one variable $\bar{f}:[0, M] \rightarrow \mathbb{R}$. We assume that $\bar{f}$ is affine on the intervals $[s-1, s], s=1, \ldots, M$. Let

$$
\bar{v}_{s}=\bar{f}(s)-\bar{f}(s-1), \quad s=1, \ldots, M .
$$

Let us note that the knowledge of the vector $\bar{v}=\left(\bar{v}_{1}, \ldots, \bar{v}_{M}\right)$ allows us to reconstruct $\bar{f}(x), x \in[0, M]$, except for the constant term $\bar{f}(0)$ :

$$
\bar{f}(x)=\bar{f}(0)+\sum_{s=1}^{l} \bar{v}_{s}+\bar{v}_{l+1}(x-l),
$$

where $l$ is such that $l \leq x<l+1$. In the context of our problem (1) with the objective function (3), $\bar{f}(\cdot)$ represents the expected value of a coordinate function, $\mathbb{E}\left\{f_{i}(\cdot)\right\}$.

The main idea of the algorithm is to recursively update a random vector $v^{k}$ taking values in $\mathbb{R}^{M}, k=1,2, \ldots$, to achieve convergence of $v^{k}$ to $\bar{v}$ (in some stochastic sense). We still denote by $(\Omega, \mathscr{F}, \mathbb{P})$ the probability space on which this sequence is defined.

Let us note that by the concavity of $\bar{f}$ the vector $\bar{v}$ has nonincreasing components:

$$
\bar{v}_{s+1} \leq \bar{v}_{s}, \quad s=1, \ldots, M-1 .
$$

We shall at first assume that there exists a constant $B$ such that

$$
\bar{v}_{1} \leq B, \quad \bar{v}_{M} \geq-B .
$$

Clearly, the set $V$ of vectors satisfying (7)-(8) is convex and closed. We shall ensure that all our approximate slopes $v^{k}$ are also elements of $V$. To this, end we shall employ the operation of orthogonal projection on $V$ :

$$
\Pi_{V}(z)=\arg \min \left\{\|v-z\|^{2}: v \in V\right\} .
$$


We show in $\S 5$ that for the set $V$ defined by (7) such a projection can be calculated in an easy way.

Our learning algorithm, which is called the separable, projective, approximation routine (SPAR), is given in Algorithm 1.

AlgoRIthm 1. Separable, projective approximation routine (SPAR).

Step 0. Set $v^{1} \in V, k=1$.

Step 1. Sample $s^{k} \in\{1, \ldots, M\}$.

Step 2. Observe a random variable $\eta^{k}$ such that

$$
\mathbb{E}\left\{\eta^{k} \mid v^{1}, \ldots, v^{k} ; s^{1}, \ldots, s^{k}\right\}=\bar{v}_{s^{k}}, \text { a.s. }
$$

Step 3. Calculate the vector $z^{k} \in \mathbb{R}^{M}$ as follows:

$$
z_{s}^{k}= \begin{cases}\left(1-\alpha_{k}\right) v_{s}^{k}+\alpha_{k} \eta^{k} & \text { if } s=s^{k}, \\ v_{s}^{k} & \text { otherwise }\end{cases}
$$

where $\alpha_{k} \in(0,1]$.

Step 4. Calculate $v^{k+1}=\Pi_{V}\left(z^{k}\right)$, increase $k$ by one, and go to Step 1 .

At this moment we shall not specify the way in which $s^{k}$ is defined, except to say that $s^{k}$ is a random variable. Specific conditions on it will be formulated later. We use $\mathscr{F}_{k}$ to denote the $\sigma$-subalgebra generated by $v^{1}, \ldots, v^{k}, s^{1}, \ldots, s^{k-1}$. We denote

$$
p_{s}^{k}=\mathbb{P}\left\{s^{k}=s \mid \mathscr{F}_{k}\right\}, \quad s=1, \ldots, M .
$$

The stepsizes $\alpha_{k}$ employed at Step 3 may also be random, but must be $\mathscr{F}_{k}$-measurable.

Let us denote by $\xi^{k}$ the random vector with the components

$$
\xi_{s}^{k}= \begin{cases}-\eta^{k}+v_{s}^{k} & \text { if } s=s^{k}, \\ 0 & \text { otherwise. }\end{cases}
$$

We can now rewrite the method compactly as

$$
v^{k+1}=\Pi_{V}\left(v^{k}-\alpha_{k} \xi^{k}\right), \quad k=1,2, \ldots
$$

It follows from (10) that

$$
\mathbb{E}\left\{\xi_{s}^{k} \mid \mathscr{F}_{k}\right\}=p_{s}^{k}\left(v_{s}^{k}-\bar{v}_{s}\right), \quad s=1, \ldots, M .
$$

Thus,

$$
\mathbb{E}\left\{\xi^{k} \mid \mathscr{F}_{k}\right\}=P^{k}\left(v^{k}-\bar{v}\right), \quad P^{k}=\operatorname{diag}\left(p_{s}^{k}\right)_{s=1}^{M} .
$$

We assume that there exists a constant $C$ such that for all $k$,

$$
\mathbb{E}\left\{\left\|\eta^{k}\right\|^{2} \mid v^{1}, \ldots, v^{k} ; s^{1}, \ldots, s^{k}\right\} \leq C \quad \text { a.s. }, \quad k=1,2, \ldots .
$$

We also assume that

$$
\begin{gathered}
\sum_{k=1}^{\infty} \alpha_{k}=\infty \quad \text { a.s., } \\
\sum_{k=1}^{\infty} \mathbb{E} \alpha_{k}^{2}<\infty, \\
\liminf _{k \rightarrow \infty} p_{s}^{k}>0 \quad \text { a.s., } \quad s=1, \ldots, M .
\end{gathered}
$$


THEOREM 1. Assume (10) and (13)-(16). Then, SPAR generates a sequence $\left\{v^{k}\right\}$ such that $v^{k} \rightarrow \bar{v}$ a.s.

PROOF. Our proof is standard, but we present it here to derive some useful inequalities that will be applied later. By the nonexpansiveness of the projection $\Pi_{V}(\cdot)$,

$$
\left\|v^{k+1}-\bar{v}\right\|^{2} \leq\left\|z^{k}-\bar{v}\right\|^{2}=\left\|v^{k}-\bar{v}\right\|^{2}-2 \alpha_{k}\left\langle v^{k}-\bar{v}, \xi^{k}\right\rangle+\alpha_{k}^{2}\left\|\xi^{k}\right\|^{2} .
$$

We add and subtract the term $2 \alpha_{k}\left\langle v^{k}-\bar{v}, P^{k}\left(v^{k}-\bar{v}\right)\right\rangle$ to obtain

$$
\begin{aligned}
\left\|v^{k+1}-\bar{v}\right\|^{2} \leq & \left\|v^{k}-\bar{v}\right\|^{2}-2 \alpha_{k}\left\langle v^{k}-\bar{v}, P^{k}\left(v^{k}-\bar{v}\right)\right\rangle \\
& -2 \alpha_{k}\left\langle v^{k}-\bar{v}, \xi^{k}-P^{k}\left(v^{k}-\bar{v}\right)\right\rangle+\alpha_{k}^{2}\left\|\xi^{k}\right\|^{2} .
\end{aligned}
$$

Let us consider the sequence

$$
S_{m}=\sum_{k=1}^{m} \alpha_{k}^{2}\left\|\xi^{k}\right\|^{2}, \quad m=1,2, \ldots,
$$

and let $S_{0}=0$. By the boundedness of $V$, and by (13) there exists a constant $C_{1}$ such that $\mathbb{E}\left\{\alpha_{m}^{2}\left\|\xi^{m}\right\|^{2} \mid \mathscr{F}_{m}\right\} \leq C_{1} \alpha_{m}^{2}$ a.s. for all $m$. Therefore, in view of the $\mathscr{F}_{m}$-measurability of $\alpha_{m}$,

$$
S_{m-1} \leq \mathbb{E}\left\{S_{m} \mid \mathscr{F}_{m}\right\} \leq S_{m-1}+C_{1} \alpha_{m}^{2}, \quad m=1,2, \ldots .
$$

Taking the expected value we obtain that $\mathbb{E}\left\{S_{m}\right\} \leq \mathbb{E}\left\{S_{m-1}\right\}+C_{1} \mathbb{E}\left\{\alpha_{m}^{2}\right\}$ for all $m$, and thus

$$
\mathbb{E}\left\{S_{m}\right\} \leq C_{1} \mathbb{E}\left\{\sum_{k=1}^{m} \alpha_{k}^{2}\right\}
$$

The last two displayed relations and Assumption (15) imply that the sequence $\left\{S_{m}\right\}$ is a submartingale, which is convergent a.s., by virtue of (Doob 1953, Theorem 4.1).

Consider now the series

$$
U_{m}=\sum_{k=1}^{m} \alpha_{k}\left\langle v^{k}-\bar{v}, \xi^{k}-P^{k}\left(v^{k}-\bar{v}\right)\right\rangle, \quad m=1,2, \ldots,
$$

and let $U_{0}=0$. By (12), $\mathbb{E}\left\{U_{m} \mid \mathscr{F}_{m}\right\}=U_{m-1}, m=1,2, \ldots$, and thus the sequence $\left\{U_{m}\right\}$ is a martingale. We can write Equation (18) as

$$
U_{m}=U_{m-1}+\alpha_{m}\left\langle v^{m}-\bar{v}, \xi^{m}-P^{m}\left(v^{m}-\bar{v}\right)\right\rangle .
$$

Squaring both sides and taking the expectation yields

$$
\begin{aligned}
\mathbb{E}\left\{U_{m}^{2} \mid \mathscr{F}_{m}\right\}= & U_{m-1}^{2}+\mathbb{E}\left\{\left[\alpha_{m}\left\langle v^{m}-\bar{v}, \xi^{m}-P^{m}\left(v^{m}-\bar{v}\right)\right\rangle\right]^{2} \mid \mathscr{F}_{m}\right\} \\
& +\mathbb{E}\left\{U_{m-1}\left(\alpha_{m}\left\langle v^{m}-\bar{v}, \xi^{m}-P^{m}\left(v^{m}-\bar{v}\right)\right\rangle\right) \mid \mathscr{F}_{m}\right\} \\
= & U_{m-1}^{2}+\mathbb{E}\left\{\left[\alpha_{m}\left\langle v^{m}-\bar{v}, \xi^{m}-P^{m}\left(v^{m}-\bar{v}\right)\right\rangle\right]^{2} \mid \mathscr{F}_{m}\right\},
\end{aligned}
$$

where the last term in Equation (19) is zero, due to (12). By the boundedness of $V$, and by (13), there exists a constant $C_{2}$ such that

$$
\mathbb{E}\left\{\left[\alpha_{m}\left\langle v^{m}-\bar{v}, \xi^{m}-P^{m}\left(v^{m}-\bar{v}\right)\right\rangle\right]^{2} \mid \mathscr{F}_{m}\right\} \leq C_{2} \alpha_{m}^{2}, \quad m=1,2, \ldots .
$$

Equations (19) and (20) yield

$$
\mathbb{E}\left\{U_{m}^{2} \mid \mathscr{F}_{m}\right\} \leq U_{m-1}^{2}+C_{2} \alpha_{m}^{2}, \quad m=1,2, \ldots .
$$


Taking the expected value, we conclude that

$$
\mathbb{E}\left\{U_{m}^{2}\right\} \leq C_{2} \mathbb{E}\left\{\sum_{k=1}^{m} \alpha_{k}^{2}\right\}, \quad m=1,2, \ldots
$$

Assumption (15) implies that the martingale $\left\{U_{m}\right\}$ is convergent a.s., by virtue of Doob 1953, Theorem 4.1. Therefore, (17) may be rewritten as

$$
\left\|v^{k+1}-\bar{v}\right\|^{2} \leq\left\|v^{k}-\bar{v}\right\|^{2}-2 \alpha_{k}\left\langle v^{k}-\bar{v}, P^{k}\left(v^{k}-\bar{v}\right)\right\rangle+A_{k},
$$

where $A_{k}=\left(S_{k}-S_{k-1}\right)-\left(U_{k}-U_{k-1}\right)$ and $\sum_{k=1}^{\infty} A_{k}$ is finite a.s. This implies that

$$
\left\|v^{k+1}-\bar{v}\right\|^{2}+\sum_{j=k+1}^{\infty} A_{j} \leq\left\|v^{k}-\bar{v}\right\|^{2}+\sum_{j=k}^{\infty} A_{j}, \quad k=1,2, \ldots
$$

The sequence $\left\|v^{k}-\bar{v}\right\|^{2}+\sum_{j=k}^{\infty} A_{j}, k=1,2, \ldots$, is nonincreasing and bounded from below a.s., and hence is convergent. Thus, the sequence $\left\{\left\|v^{k}-\bar{v}\right\|^{2}\right\}$ is convergent a.s. From (21) we get

$$
\sum_{k=1}^{\infty} \alpha_{k}\left\langle v^{k}-\bar{v}, P^{k}\left(v^{k}-\bar{v}\right)\right\rangle<\infty \quad \text { a.s. }
$$

Using (14) and (16) we deduce that a.s. there must exist an infinite subset of indices $\mathscr{K} \subseteq \mathbb{N}$ and a subsequence $\left\{v^{k}\right\}, k \in \mathscr{K}$, such that $v^{k} \rightarrow \bar{v}$ for $k \in \mathscr{K}$. Because the sequence of distances $\left\|v^{k}-\bar{v}\right\|^{2}$ is convergent, the entire sequence $\left\{v^{k}\right\}$ converges to $\bar{v}$.

If we remove Inequalities (8) from the definition of $V$, only small technical changes are needed to ensure convergence a.s. Instead of the steps $\alpha_{k} \xi^{k}$ we need to use normalized steps $\alpha_{k} \gamma_{k} \xi^{k}$, where the normalizing coefficients have the form

$$
\gamma_{k}=\left(\max \left(\left\|v^{k}\right\|, B\right)\right)^{-1}
$$

for some large constant $B$. We first prove that both martingales $\left\{S_{m}\right\}$ and $\left\{U_{m}\right\}$ converge, due to the damping by the $\gamma_{k}$ s. Then, the corresponding version of (21) yields the boundedness of $\left\{v^{k}\right\}$ a.s. Consequently, the normalizing coefficients are bounded away from 0 a.s., and the remaining part of the analysis goes through, as well. In our further considerations we shall still assume that Inequalities (8) are present in the definition of $V$ to avoid unnecessary notational complications associated with the normalizing coefficients $\gamma_{k}$.

In many applications, at a given point $s^{k} \in\{1, \ldots, M-1\}$, we can observe two random variables: $\eta^{k}$ satisfying (10), and $\eta_{+}^{k}$ such that

$$
\mathbb{E}\left\{\eta_{+}^{k} \mid v^{1}, \ldots, v^{k} ; s^{1}, \ldots, s^{k}\right\}=\bar{v}_{s^{k}+1}
$$

and

$$
\mathbb{E}\left\{\left\|\eta_{+}^{k}\right\|^{2} \mid v^{1}, \ldots, v^{k} ; s^{1}, \ldots, s^{k}\right\} \leq C, \quad k=1,2, \ldots
$$

This was illustrated in Example 1.

Our algorithm can be easily adapted to this case, too. The only difference is Step 3, where we use both random observations, whenever they are available:

$$
z_{s}^{k}= \begin{cases}\left(1-\alpha_{k}\right) v_{s}^{k}+\alpha_{k} \eta^{k} & \text { if } s=s^{k} \\ \left(1-\alpha_{k}\right) v_{s}^{k}+\alpha_{k} \eta_{+}^{k} & \text { if } s^{k}<M \text { and } s=s^{k}+1 \\ v_{s}^{k} & \text { otherwise. }\end{cases}
$$


The analysis of this version of the method is similar to the basic case. We define

$$
\xi_{s}^{k}= \begin{cases}-\eta^{k}+v_{s}^{k} & \text { if } s=s^{k} \\ -\eta_{+}^{k}+v_{s}^{k} & \text { if } s^{k}<M \text { and } s=s^{k}+1 \\ 0 & \text { otherwise. }\end{cases}
$$

It follows from (10) and (22) that

$$
\mathbb{E}\left\{\xi_{s}^{k} \mid \mathscr{F}_{k}\right\}= \begin{cases}p_{s}^{k}\left(v_{s}^{k}-\bar{v}_{s}\right) & \text { if } s=1, \\ \left(p_{s}^{k}+p_{s-1}^{k}\right)\left(v_{s}^{k}-\bar{v}_{s}\right) & \text { if } 1<s \leq M .\end{cases}
$$

Therefore, after replacing the coefficients $p_{s}^{k}$ by

$$
\bar{p}_{s}^{k}= \begin{cases}p_{s}^{k} & \text { if } s=1, \\ p_{s}^{k}+p_{s-1}^{k} & \text { if } 1<s \leq M,\end{cases}
$$

we can reduce this version of the method to the basic case analyzed earlier.

3. Using objective value observations. In the applications that we have in mind, our observations provide us with more information than just the estimate of the slope of the objective function at $s^{k}$. We also observe the value of the objective function at $s^{k}$ corresponding to some outcome $\omega^{k}$. We denote this by

$$
\theta^{k}=f\left(s^{k}, \omega^{k}\right) .
$$

Usually, we know the value of $\bar{f}(0)$ and with no loss of generality we assume that $\bar{f}(0)=0$. We also assume that $\theta^{k}$ satisfies

$$
\mathbb{E}\left\{\theta^{k} \mid v^{1}, \ldots, v^{k} ; s^{1}, \ldots, s^{k}\right\}=\bar{f}\left(s^{k}\right)=\sum_{i=1}^{s^{k}} \bar{v}_{i} \quad \text { a.s. }
$$

Condition (26) is trivially satisfied when $\omega^{k}$ is independent of $v^{1}, \ldots, v^{k} ; s^{1}, \ldots, s^{k}$, but this does not necessarily have to be the case. We can now use $\left\{\theta^{k}\right\}$ to facilitate the convergence to $\bar{v}$. The algorithm is described in Algorithm 2.

Algorithm 2. SPAR with objective function updates (SPAR-Obj).

Step 0 . Set $v^{1} \in V, k=1$.

Step 1. Sample $s^{k} \in\{1, \ldots, M\}$.

Step 2. Observe random variables $\eta^{k}$ and $\theta^{k}$ satisfying (10) and (26).

Step 3. Calculate the vector $z^{k} \in \mathbb{R}^{M}$ as follows:

$$
z_{s}^{k}= \begin{cases}v_{s}^{k}+\alpha_{k}\left(\theta^{k}-\sum_{i=1}^{s^{k}} v_{i}^{k}\right) & \text { for } s=1, \ldots, s^{k}-1, \\ \left(1-\alpha_{k}\right) v_{s}^{k}+\alpha_{k} \eta^{k}+\alpha_{k}\left(\theta^{k}-\sum_{i=1}^{s^{k}} v_{i}^{k}\right) & \text { for } s=s^{k}, \\ v_{s}^{k} & \text { otherwise, }\end{cases}
$$

where $\alpha_{k} \in(0,1]$.

Step 4. Calculate $v^{k+1}=\Pi_{V}\left(z^{k}\right)$, increase $k$ by one, and go to Step 1 .

We additionally assume that there exists a constant $C$ such that

$$
\mathbb{E}\left\{\left(\theta^{k}\right)^{2} \mid v^{1}, \ldots, v^{k} ; s^{1}, \ldots, s^{k}\right\} \leq C, \quad k=1,2, \ldots .
$$

We have a result similar to Theorem 1 . 
THEOREm 2. Assume (10), (13)-(16), (26), and (28). Then, SPAR-Obj generates a sequence $\left\{v^{k}\right\}$ such that $v^{k} \rightarrow \bar{v}$ a.s.

Proof. Let us calculate the conditional expectation of the vector $\xi^{k}=\left(v^{k}-z^{k}\right) / \alpha_{k}$. Directly from (10) and (26) we have

$$
\left(\mathbb{E}\left\{\xi^{k} \mid \mathscr{F}_{k}\right\}\right)_{s}= \begin{cases}\sum_{i=1}^{s^{k}}\left(v_{i}^{k}-\bar{v}_{i}^{k}\right) & \text { for } s=1, \ldots, s^{k}-1, \\ v_{s^{k}}^{k}-\bar{v}_{s^{k}}^{k}+\sum_{i=1}^{s^{k}}\left(v_{i}^{k}-\bar{v}_{i}^{k}\right) & \text { for } s=s^{k}, \\ 0 & \text { for } s=s^{k}+1, \ldots, M .\end{cases}
$$

Therefore,

$$
\begin{aligned}
\left(\mathbb{E}\left\{\xi^{k} \mid \mathscr{F}_{k}\right\}\right)_{j}= & p_{j}^{k}\left(v_{j}^{k}-\bar{v}_{j}\right)+\sum_{s=j}^{M} p_{s}^{k} \sum_{i=1}^{s}\left(v_{i}^{k}-\bar{v}_{i}^{k}\right) \\
= & \sum_{i=1}^{j-1}\left(\sum_{s=j}^{M} p_{s}^{k}\right)\left(v_{i}^{k}-\bar{v}_{i}^{k}\right)+\left(p_{j}^{k}+\sum_{s=j}^{M} p_{s}^{k}\right)\left(v_{j}^{k}-\bar{v}_{j}^{k}\right) \\
& +\sum_{i=j+1}^{M}\left(\sum_{s=i}^{M} p_{s}^{k}\right)\left(v_{i}^{k}-\bar{v}_{i}^{k}\right) .
\end{aligned}
$$

Consider the matrix $W^{k}$ of dimension $M \times M$, with the entries

$$
w_{i j}^{k}= \begin{cases}\sum_{s=j}^{M} p_{s}^{k} & \text { if } i \leq j, \\ \sum_{s=i}^{M} p_{s}^{k} & \text { if } i>j .\end{cases}
$$

The last two relations yield

$$
\mathbb{E}\left\{\xi^{k} \mid \mathscr{F}_{k}\right\}=\left(P^{k}+W^{k}\right)\left(v^{k}-\bar{v}^{k}\right),
$$

where $P^{k}=\operatorname{diag}\left(p_{j}^{k}\right)_{j=1}^{M}$. We have

$$
W^{k}=\sum_{l=1}^{M} W_{l}^{k},
$$

where each of the matrices $W_{l}^{k}$ has entries

$$
\left(W_{l}^{k}\right)_{i j}= \begin{cases}p_{l}^{k} & \text { if } i, j \leq l, \\ 0 & \text { otherwise }\end{cases}
$$

Each $W_{l}^{k}$ is positive semidefinite, and thus $W^{k}$ is also positive semidefinite. Now we can proceed as in the proof of Theorem 1. We have an inequality similar to (17):

$$
\begin{aligned}
\left\|v^{k+1}-\bar{v}\right\|^{2} \leq & \left\|v^{k}-\bar{v}\right\|^{2}-2 \alpha_{k}\left\langle v^{k}-\bar{v},\left(P^{k}+W^{k}\right)\left(v^{k}-\bar{v}\right)\right\rangle \\
& -2 \alpha_{k}\left\langle v^{k}-\bar{v}, \xi^{k}-\left(P^{k}+W^{k}\right)\left(v^{k}-\bar{v}\right)\right\rangle+\alpha_{k}^{2}\left\|\xi^{k}\right\|^{2} .
\end{aligned}
$$

Using (29) and (15) we can rewrite this inequality as

$$
\left\|v^{k+1}-\bar{v}\right\|^{2} \leq\left\|v^{k}-\bar{v}\right\|^{2}-2 \alpha_{k}\left\langle v^{k}-\bar{v},\left(P^{k}+W^{k}\right)\left(v^{k}-\bar{v}\right)\right\rangle+A_{k},
$$

where $\sum_{k=1}^{\infty} A_{k}$ is finite a.s. Because $W^{k}$ is positive semidefinite, we can omit it in the above inequality and obtain (21) again. The rest of the proof is the same as in the proof of Theorem 1 . 
SPAR-Obj can be modified to assign different weights to the components of the direction associated with the observations $\eta^{k}$ and $\theta^{k}$. In particular, we may set at Step 3

$$
z_{s}^{k}= \begin{cases}v_{s}^{k}+\frac{\alpha_{k}}{\rho_{k}}\left(\theta^{k}-\sum_{i=1}^{s^{k}} v_{i}^{k}\right) & \text { for } s=1, \ldots, s^{k}-1, \\ \left(1-\alpha_{k}\right) v_{s}^{k}+\alpha_{k} \eta^{k}+\frac{\alpha_{k}}{\rho_{k}}\left(\theta^{k}-\sum_{i=1}^{s^{k}} v_{i}^{k}\right) & \text { for } s=s^{k} \\ v_{s}^{k} & \text { otherwise, }\end{cases}
$$

where $0<\rho^{\min } \leq \rho_{k} \leq \rho^{\max }$ and $\rho_{k}$ is $\mathscr{F}_{k}$-measurable. The analysis of this version is identical to the proof of Theorem 2. Our numerical experiments reported in $\$ 6$ indicate that the additional scaling in (30) is useful.

4. Multidimensional problems and learning while optimizing. Let us now return to Problem (1). If the next observation point $s^{k}=\left(s_{1}^{k}, \ldots, s_{n}^{k}\right)$ is sampled at random, and if for each coordinate $i$ the probabilities

$$
p_{i s}^{k}=\mathbb{P}\left\{s_{i}^{k}=s \mid \mathscr{F}_{k}\right\}, \quad s=1, \ldots, M,
$$

satisfy Assumption (16), then all results of the preceding two sections apply componentwise.

The situation is different if we generate the next observation point $s^{k}$ by solving the approximate Problem (6), that is

$$
s_{i}^{k}=x_{i}^{k}, \quad i=1, \ldots, n, \quad k=1,2, \ldots .
$$

If the solution $x^{k}$ is not unique, we choose it at random from the set of optimal solutions of (6). For each coordinate function $f_{i}$, we observe two random variables: $\eta_{i}^{k}$ satisfying (10) and $\eta_{i+}^{k}$ satisfying (22). Then, we update the left and right slopes for each function $f_{i}$ according to (24), and the iteration continues. In this way we define the sequences $\left\{v_{i}^{k}\right\}$, $k=1,2, \ldots$, of estimates of the slopes of $f_{i}, i=1, \ldots, n$, and a sequence $x^{k}$ of the solutions of approximate models (6).

This algorithm is well defined if the approximate Problems (6) have integer solutions for all concave piecewise linear functions $f_{i}$ having integer break points. This is true, for example, for models having alternative network representations, such as those discussed in Powell and Topaloglu (2003).

Note that in the previous two sections our convergence proofs depend on the assumption that $\liminf _{k \rightarrow \infty} p_{i s}^{k}>0$ a.s. for $s=1, \ldots, M, i=1, \ldots, n$. However, when $s^{k}$ is selected as $s^{k}=\arg \max _{x \in X} \sum_{i=1}^{n} \bar{f}_{i}^{k}\left(x_{i}\right)$, this assumption may not be satisfied. In this section, we show that even with this new choice of $s^{k}$, the sequence $\left\{s^{k}\right\}$ converges to an optimal solution of $\max _{x \in X} \sum_{i=1}^{n} \bar{f}_{i}\left(x_{i}\right)$, provided a certain stability condition is satisfied.

Let us note that for a concave, piecewise linear, and separable function

$$
F(x)=\sum_{i=1}^{n} f_{i}\left(x_{i}\right)
$$

where each $f_{i}$ is defined as

$$
f_{i}\left(x_{i}\right)=\sum_{s=1}^{l} v_{s}+v_{l+1}(x-l),
$$

with an integer $l$ such that $l \leq x<l+1$, the subdifferential of $F$ at an integer point $x$ is given by

$$
\partial F(x)=\left[v_{1, x_{1}+1}, v_{1, x_{1}}\right] \times\left[v_{2, x_{2}+1}, v_{2, x_{2}}\right] \times \cdots \times\left[v_{n, x_{n}+1}, v_{n, x_{n}}\right] .
$$


Under the Slater constraint qualification, the necessary and sufficient condition of optimality for problem

$$
\max _{x \in X} F(x),
$$

where $X$ is a convex closed set, has the form

$$
0 \in \partial F(x)-N(x),
$$

with $N(x)$ being the normal cone to $X$ at $x$. An optimal point $\hat{x}$ is called stable if it satisfies

$$
0 \in \operatorname{int}[\partial F(\hat{x})-N(\hat{x})] .
$$

It can be seen directly from Conditions (33) and (34) that a stable point $\hat{x}$ is also a solution to a perturbed problem

$$
\max _{x \in X} \tilde{F}(x),
$$

provided that $\operatorname{dist}(\partial F(\hat{x}), \partial \tilde{F}(\hat{x}))<\epsilon$ and $\epsilon$ is a sufficiently small positive number.

Clearly, the solutions $x^{k}$ of our approximate Problems (6) satisfy Condition (33) for the approximate functions $f^{k}$ constructed by the method. Then, by passing to the limit, we can conclude that each accumulation point $\left(x^{*}, v^{*}\right)$ of the sequence $\left\{\left(x^{k}, v^{k}\right)\right\}$ satisfies the condition

$$
0 \in \partial F^{*}\left(x^{*}\right)-N\left(x^{*}\right)
$$

with $F^{*}$ constructed from $v^{*}$ as in (31)-(32). We shall show that if such an accumulation point satisfies the condition of stability, it is optimal for the original problem.

Theorem 3. Assume that for each $i=1, \ldots, n$, Conditions (10), (13)-(15), and (22)-(23) are satisfied. If an accumulation point $\left(x^{*}, v^{*}\right)$ of the sequence $\left\{\left(x^{k}, v^{k}\right)\right\}$ generated by the algorithm satisfies the stability condition

$$
0 \in \operatorname{int}\left[\partial F^{*}\left(x^{*}\right)-N\left(x^{*}\right)\right]
$$

then with probability one $x^{*}$ is an optimal solution of (1).

Proof. Let us observe that Relation (25) holds for each coordinate $i$. Therefore, Inequality (21) is true for each coordinate $i$ :

$$
\left\|v_{i}^{k+1}-\bar{v}_{i}\right\|^{2} \leq\left\|v_{i}^{k}-\bar{v}_{i}\right\|^{2}-2 \alpha_{k}\left\langle v_{i}^{k}-\bar{v}_{i}, P_{i}^{k}\left(v_{i}^{k}-\bar{v}_{i}\right)\right\rangle+A_{i k} .
$$

The matrix $P_{i}^{k}$, which is $\mathscr{F}_{k}$-measurable, is a nonnegative diagonal matrix with positive entries corresponding to the $i$ th coordinates of possible solutions to (6). Proceeding exactly as in the proof of Theorem 1, we conclude that the series $\sum_{k=1}^{\infty} A_{i k}$ is convergent a.s. Furthermore, the sequence $\left\{\left\|v_{i}^{k}-\bar{v}_{i}\right\|\right\}$ is convergent a.s. for every $i=1, \ldots, n$.

Our proof will analyze properties of sample paths of the random sequence $\left\{\left(v^{k}, x^{k}\right)\right\}$ for all elementary events $\omega \in \Omega \backslash \Omega_{0}$, where $\Omega_{0}$ is a null set. It will become clear in the course of the proof what this null set is.

Let us fix $\omega \in \Omega$ and consider a convergent subsequence $\left\{\left(v^{k}(\omega), x^{k}(\omega)\right)\right\}, k \in \mathscr{K}(\omega)$, where $\mathscr{K}(\omega) \subseteq \mathbb{N}$ is some infinite set of indices. Let us denote by $\left(v^{*}, x^{*}\right)$ the limit of this subsequence. This limit depends on $\omega$ too, but we shall omit the argument $\omega$ to simplify notation.

If the stability condition holds, then there exists $\epsilon>0$ such that for all iterations $k$ for which $\left|v_{i, x_{i}^{*}}^{k}(\omega)-v_{i, x_{i}^{*}}^{*}\right| \leq \epsilon, i=1, \ldots, n$, the solution $x^{k}$ of the approximate Problem (6) is equal to $x^{*}$. Then, the coefficients $p_{i, s}^{k}$ are equal to 1 for $s=x_{i}^{*}$ and $s=x_{i}^{*}+1$, and are zero otherwise, for each function $i$. Let us fix an arbitrary $i$ and focus our attention on the points $s=x_{i}^{*}$. Inequality (36) implies

$$
\left\|v_{i}^{k+1}(\omega)-\bar{v}_{i}\right\|^{2} \leq\left\|v_{i}^{k}(\omega)-\bar{v}_{i}\right\|^{2}-2 \alpha_{k}(\omega)\left(v_{i, x_{i}^{*}}^{k}(\omega)-\bar{v}_{i, x_{i}^{*}}\right)^{2}+A_{i k}(\omega) .
$$


The series $\sum_{k} A_{i k}$ is convergent a.s. Let $k \in \mathscr{K}(\omega)$ be large enough that $\left|v_{i, x_{i}^{*}}^{k}(\omega)-v_{i, x_{i}^{*}}^{*}\right|<$ $\epsilon / 2$. Consider $j \geq k$ such that

$$
\left|v_{i, x_{i}^{*}}^{j}(\omega)-v_{i, x_{i}^{*}}^{*}\right| \leq \epsilon \text { for all } i=1, \ldots, n .
$$

Let us suppose that the $i$ th coordinate of the limit point is not optimal, i.e.,

$$
v_{i, x_{i}^{*}}^{*} \neq \bar{v}_{i, x_{i}^{*}}
$$

We shall prove that it leads to a contradiction. The remaining part of our proof has three stages.

Stage 1 . We can always choose a sufficiently small $\epsilon>0$ such that $\left|v_{i, x_{i}^{*}}^{*}-\bar{v}_{i, x_{i}^{*}}\right|>2 \epsilon$. Then for the iterations $j$ satisfying (38) we have $\left|v_{i, x_{i}^{*}}^{j}(\omega)-\bar{v}_{i, x_{i}^{*}}\right|>\epsilon$, and Inequality (37) implies

$$
\left\|v_{i}^{j+1}(\omega)-\bar{v}_{i}\right\|^{2} \leq\left\|v_{i}^{j}(\omega)-\bar{v}_{i}\right\|^{2}-2 \alpha_{j}(\omega) \epsilon^{2}+A_{i j}(\omega) .
$$

The series $\sum_{j} \alpha_{j}(\omega)$ is divergent and the series $\sum_{j} A_{i j}(\omega)$ is convergent, unless $\omega$ is in a certain null set. If the set of consecutive $j \geq k$ for which Condition (38) holds was infinite, Inequality (40) would lead to a contradiction. Therefore, for all $k \in \mathscr{K}(\omega)$ and all sufficiently small $\epsilon>0$, the random index

$$
l(k, \epsilon, \omega)=\inf \left\{j \geq k: \max _{1 \leq i \leq n}\left|v_{i, x_{i}^{*}}^{j}(\omega)-v_{i, x_{i}^{*}}^{*}\right|>\epsilon\right\}
$$

is finite.

Stage 2. We shall prove that the sum of stepsizes between $k \in \mathscr{K}(\omega)$ and $l(k, \epsilon, \omega)-1$ is at least of order $\epsilon$ if $k$ is large enough. By the definition of $l(k, \epsilon, \omega)$ we have, for some $i$, $\left|v_{i, x_{i}^{*}}^{l(k, \omega)}(\omega)-v_{i, x_{i}^{*}}^{*}\right|>\epsilon$. Because $v^{k}(\omega) \rightarrow v^{*}, k \in \mathscr{K}(\omega)$, we also have $\left\|v_{i}^{l(k, \epsilon, \omega)}-v_{i}^{k}(\omega)\right\|>$ $\epsilon / 2$ for all sufficiently large $k \in \mathscr{R}(\omega)$. Thus,

$$
\sum_{j=k}^{l(k, \epsilon, \omega)-1} \alpha_{j}(\omega)\left\|\xi_{i}^{j}(\omega)\right\|>\epsilon / 2 .
$$

Let us observe that Conditions (13) and (15) imply that for each $i$ the random series

$$
\sum_{k=1}^{\infty} \alpha_{k}\left(\left\|\xi_{i}^{k}\right\|-\mathbb{E}\left\{\left\|\xi_{i}^{k}\right\| \mid \mathscr{F}_{k}\right\}\right)
$$

is a convergent martingale. Therefore, unless $\omega$ is in a certain null set,

$$
\sum_{j=k}^{l(k, \epsilon, \omega)-1} \alpha_{j}(\omega)\left\|\xi_{i}^{j}(\omega)\right\|=\sum_{j=k}^{l(k, \epsilon, \omega)-1} \alpha_{j}(\omega) \kappa_{i j}(\omega)+\sigma_{i k}(\omega),
$$

where $\kappa_{i j}=\mathbb{E}\left\{\left\|\xi_{i}^{j}\right\| \mid \mathscr{F}_{j}\right\}$ and $\sigma_{i k}(\omega)=\sum_{j=k}^{l(k, \epsilon, \omega)-1} \alpha_{j}(\omega)\left(\left\|\xi_{i}^{j}(\omega)\right\|-\kappa_{i j}(\omega)\right) \rightarrow 0$ as $k \rightarrow \infty$, $k \in \mathscr{K}(\omega)$. This, combined with (41), implies that for all sufficiently large $k \in \mathscr{K}(\omega)$,

$$
\sum_{j=k}^{l(k, \epsilon, \omega)-1} \alpha_{j}(\omega) \kappa_{i j}(\omega) \geq \epsilon / 3 .
$$

From Assumption (13) it follows that there exists a constant $C$ such that $\kappa_{i j}(\omega) \leq C$ for all $i$ and $j$. Using this in the last displayed inequality we obtain

$$
\sum_{j=k}^{l(k, \epsilon, \omega)-1} \alpha_{j}(\omega) \geq \frac{\epsilon}{3 C}
$$

for all sufficiently small $\epsilon>0$ and all sufficiently large $k \in \mathscr{K}(\omega)$. 
Stage 3. Summing (40) from $k$ to $l(k, \epsilon, \omega)-1$, letting $\delta=1 / 3 C$, and combining with (42) gives, for some coordinate $i$,

$$
\left\|v_{i}^{l(k, \epsilon, \omega)}(\omega)-\bar{v}_{i}\right\|^{2} \leq\left\|v_{i}^{k}(\omega)-\bar{v}_{i}\right\|^{2}-2 \delta \epsilon^{3}+\sum_{j=k}^{l(k, \epsilon, \omega)-1} A_{i j}(\omega) .
$$

Let $\Delta(\omega)$ be the limit of the entire sequence $\left\{\left\|v_{i}^{j}(\omega)-\bar{v}_{i}\right\|^{2}\right\}$ as $j \rightarrow \infty$, whose existence has been established at the beginning of the proof. Passing to the limit with $k \rightarrow \infty, k \in \mathscr{K}(\omega)$, and using the fact that $\sum_{j=k}^{\infty} A_{i j}(\omega) \rightarrow 0$ as $k \rightarrow \infty$, we obtain

$$
\Delta(\omega) \leq \Delta(\omega)-2 \delta \epsilon^{3},
$$

a contradiction. Therefore, our Assumption (39) must be false, and we have

$$
v_{i, x_{i}^{*}}^{*}=\bar{v}_{i, x_{i}^{*}} \text { for all } i=1, \ldots, n .
$$

Inequality (37) is also true with $x_{i}^{k}$ replaced by $x_{i}^{k}+1$ (if $x_{i}^{k}<M_{i}$ ). We can thus apply the same argument to prove

$$
v_{i, x_{i}^{*}+1}^{*}=\bar{v}_{i, x_{i}^{*}+1} \text { for all } i=1, \ldots, n .
$$

For $x_{i}^{*}=M_{i}$, we take the convention that $v_{i, x_{i}^{*}+1}^{*}=\bar{v}_{i, x_{i}^{*}+1}=-\infty$. Consequently,

$$
\partial F\left(x^{*}\right)=\partial F^{*}\left(x^{*}\right)
$$

and the point $x^{*}$ is optimal for (1).

The assumptions of Theorem 3 are stronger than those of Theorems 1 and 2. However, the result of Theorem 3 is much stronger. For a general closed convex set $X$, it may be very costly to devise a sampling scheme for $\left\{s^{k}\right\}$ satisfying $\liminf _{k \rightarrow \infty} p_{i s}^{k}>0$ a.s. for $s=$ $1, \ldots, M, i=1, \ldots, n$. Theorem 3 saves us from devising such a sampling scheme and lets us pick $s^{k}$ by simply solving an optimization problem. The stability Assumption (35) is difficult to verify a priori, but it is very easy to check a posteriori, when the accumulation point $x^{*}$ and the approximate function $F^{*}$ have been identified.

In a similar way (and under identical assumptions) we can prove the convergence of the version that uses function value estimates.

Theorem 4. Assume (10), (13)-(15), (22)-(23), (26), and (28). If an accumulation point $\left(x^{*}, v^{*}\right)$ of the sequence $\left\{\left(x^{k}, v^{k}\right)\right\}$ generated by SPAR-Obj satisfies the stability Condition (35), then with probability one $x^{*}$ is an optimal solution of (1).

The proof is almost a verbatim copy of the proof of Theorem 3, with the modifications as in Theorem 2.

5. Projection. Let us now describe the way the projection $v=\Pi_{V}(z)$ can be calculated. Clearly, $v$ is the solution to the quadratic programming problem

$$
\begin{gathered}
\min \frac{1}{2}\|v-z\|^{2} \\
\text { subject to } \quad v_{s+1}-v_{s} \leq 0, \quad s=0, \ldots, M,
\end{gathered}
$$

where for uniformity we denote $v_{0}=B$, and $v_{M+1}=-B$. Associating with (46) Lagrange multipliers $\lambda_{s} \geq 0, s=0, \ldots, M$, we obtain the necessary and sufficient optimality conditions

$$
\begin{gathered}
v_{s}=z_{s}+\lambda_{s}-\lambda_{s-1}, \quad s=1, \ldots, M, \\
\lambda_{s}\left(v_{s+1}-v_{s}\right)=0, \quad s=0, \ldots, M .
\end{gathered}
$$


If $i_{1}, \ldots, i_{2}$ is a sequence of coordinates such that

$$
v_{i_{1}-1}>v_{i_{1}}=v_{i_{1}+1}=\cdots=c=\cdots=v_{i_{2}-1}=v_{i_{2}}>v_{i_{2}+1},
$$

then adding Equations (47) from $i_{1}$ to $i_{2}$ yields

$$
c=\frac{1}{i_{2}-i_{1}+1} \sum_{s=i_{1}}^{i_{2}} z_{s}
$$

If $i_{1}=1$, then $c$ is the minimum of the above average and $B$, and for $i_{2}=M$ the maximum of $-B$, and this average has to be taken.

The second useful observation is that $v^{k} \in V$ and $z^{k}$ computed by (11) differs from $v^{k}$ in just one coordinate. If $z^{k} \notin V$, one of two cases must occur: Either $z_{s^{k}-1}^{k}<z_{s^{k}}^{k}$ or $z_{s^{k}+1}^{k}>z_{s^{k}}^{k}$.

If $z_{s^{k}-1}^{k}<z_{s^{k}}^{k}$, we search for the largest $1<i \leq s^{k}$ for which

$$
z_{i-1}^{k} \geq \frac{1}{s^{k}-i+1} \sum_{s=i}^{s^{k}} z_{s}^{k}
$$

If such $i$ cannot be found, we set $i=1$. Then, we calculate

$$
c=\frac{1}{s^{k}-i+1} \sum_{s=i}^{s^{k}} z_{s}^{k}
$$

and set

$$
v_{j}^{k+1}=\min (B, c), \quad j=i, \ldots, s^{k} .
$$

We have $\lambda_{0}=\max (0, c-B)$ and

$$
\lambda_{s}= \begin{cases}0, & s=1, \ldots, i-1, \\ \lambda_{s-1}+z_{s}-v_{s}, & s=i, \ldots, s^{k}-1, \\ 0, & s=s^{k}, \ldots, M .\end{cases}
$$

It is straightforward to verify that the solution found and the above Lagrange multipliers satisfy Conditions (47)-(48).

The procedure in the case when $z_{s^{k}}^{k}<z_{s^{k}+1}^{k}$ is symmetrical it is the same procedure applied to the graph of $z$ rotated by $\pi$.

Let us now consider the method which employs two random variables at each iteration, with Step 3 as in (24). Then, both $z_{s^{k}}$ and $z_{s^{k}+1}$ may differ from $v_{s^{k}}$ and $v_{s^{k}+1}$ (although we still have $z_{s^{k}}>z_{s^{k}+1}$ ). We shall show that the algorithms defined by (49)-(51) can easily be adapted to this case.

Suppose that $z_{s^{k}}>z_{s^{k}-1}$. We apply (49)-(50) to compute the candidate value for $c$. Now two cases may occur.

Case 1. If $c \geq z_{s^{k}+1}$, we may apply (51). We can now focus on the points to the right: $z_{s^{k}+1}, z_{s^{k}+2}, \ldots$ We apply the symmetric analogue of (49)-(51) to these values, and the projection is accomplished.

Case 2. If $c<z_{s^{k}+1}$, the value of $c$ is not correct. We need to incorporate $z_{s^{k}+1}$ into the averaging procedure. Thus, we repeat (49)-(51) but with $s^{k}$ replaced by $s^{k}+1$ in (49), although we still search for $1<i \leq s^{k}$. After this we apply (51) for $j=i, \ldots, s^{k}+1$.

If $z_{s^{k}+1}<z_{s^{k}+2}$, the situation is symmetrical to the one discussed above (after rotating the graph of $z$ by $\pi$ ) and an analogous procedure can be applied. 
6. Experiments for separable problems. To illustrate the behavior of the methods discussed, we consider the problem in Example 1:

$$
\max _{x \in X} \mathbb{E} \sum_{i=1}^{n} f_{i}\left(x_{i}, D_{i}\right), \quad \text { where } X \text { is given in (5) and } f_{i}\left(x_{i}, D_{i}\right)=q_{i} \min \left(x_{i}, D_{i}\right)-c_{i} x_{i} .
$$

Clearly, both SPAR and SPAR-Obj can be applied to this problem componentwise: We approximate $\mathbb{E} \sum_{i=1}^{n} f_{i}\left(x_{i}, D_{i}\right)$ by $\sum_{i=1}^{n} f_{i}^{k}\left(x_{i}\right)$ at iteration $k$. For a given resource allocation $s^{k}=\left(s_{1}^{k}, \ldots, s_{n}^{k}\right)$ among $n$ activities and a sampled demand realization $D^{k}=\left(D_{1}^{k}, \ldots, D_{n}^{k}\right)$, we can separately apply the updates of SPAR and SPAR-Obj for each component $i=$ $1, \ldots, n$. In the description below, $s_{i}^{k}$ plays the role of $s^{k}$ for the $i$ th component, $\rho_{k i}$ the role of $\rho_{k}$, and so on. We compare the following methods:

- SPAR-This is the basic learning algorithm with projection.

- SPAR-Obj(a) with $\rho_{k i}=s_{i}^{k}$-This uses objective function estimates to help with the learning, using weights of $1 / s^{k}$ for the objective function estimates.

- SPAR-Obj(b) with $\rho_{k}=M_{i} s_{i}^{k}$-Same as above, but with a much smaller weight on the objective function.

- The leveling method of Topaloglu and Powell (2003) - This algorithm maintains concavity by forcing slopes that violate an updated estimate to be no larger (to the left) or no smaller (to the right) than the most recently updated cell. This algorithm has been shown to be convergent.

- The CAVE algorithm of Godfrey and Powell (2001) — This was the first algorithm suggested for adaptively estimating piecewise linear functions while maintaining concavity.

In the first series of experiments, the random variable $s^{k}$ is generated from the uniform distribution over the rectangle $\left[1, M_{1}\right] \times \cdots \times\left[1, M_{n}\right]$. We assume that each component of the demand variable $D^{k}$ is independent and Poisson distributed. Having sampled $s_{i}^{k}$ and $D_{i}^{k}$, we compute the left-hand slope of $f_{i}\left(\cdot, D_{i}^{k}\right)$ at $s_{i}^{k}$ as

$$
\eta_{i}^{k}= \begin{cases}q_{i}-c_{i} & \text { if } s_{i}^{k} \leq D_{i}^{k}, \\ -c_{i} & \text { if } s_{i}^{k}>D_{i}^{k} .\end{cases}
$$

Having obtained this slope information and the value of $s_{i}^{k}$ for iteration $k$, we can obtain the approximation $f_{i}^{k+1}$ using any of the methods mentioned above. We call this a learning step, because in this iteration we are exploring the slope of the function at randomly sampled points.

To estimate the quality of an approximation we find $\bar{x}^{k} \in \arg \max _{x \in X} \sum_{i=1}^{n} f_{i}^{k}\left(x_{i}\right)$ and compute $\mathbb{E} \sum_{i=1}^{n} f_{i}\left(\bar{x}_{i}^{k}, D_{i}\right)$. This gives us an idea about the average actual performance of the solution given by the approximation $\sum_{i=1}^{n} f_{i}^{k}$.

For each method and coordinate (activity) the sequence of step sizes is $\alpha_{k}=20 /(40+k)$. We take the number of coordinates to be $n=90 . M_{i}$ ranges between 20 and 40 , and $c_{i}$ ranges between 0.6 and 1.4 for different 90 activities. $D_{i}^{k}$ is truncated-Poisson distributed with mean ranging between 9 and 21 for $i=1, \ldots, 90$. Finally, $q_{i}=2$ for all $i=1, \ldots, n$ and $b=950$. We run each method 50 times using 100 demand realizations at each run, and Figure 1(a) presents the averages over these 50 runs.

We see that our basic learning method, SPAR, performs very well. Its quality can be slightly improved by using objective function estimates as in SPAR-Obj, but the weight associated with them must be significantly smaller than the weight associated with the slope observations, as the comparison of SPAR-Obj(a) and SPAR-Obj(b) shows.

The second series of experiments differs from the first by the method we utilized to generate the random variables $s^{k}$. Now, $s^{k}$ is chosen to be the maximizer of $\sum_{i=1}^{n} f_{i}^{k}$, as discussed in $\S 4$. We call this an optimizing step because this step involves maximizing the current approximation as opposed to selecting $s^{k}$ randomly over the interval $\left[1, M_{1}\right] \times \cdots \times\left[1, M_{n}\right]$. 


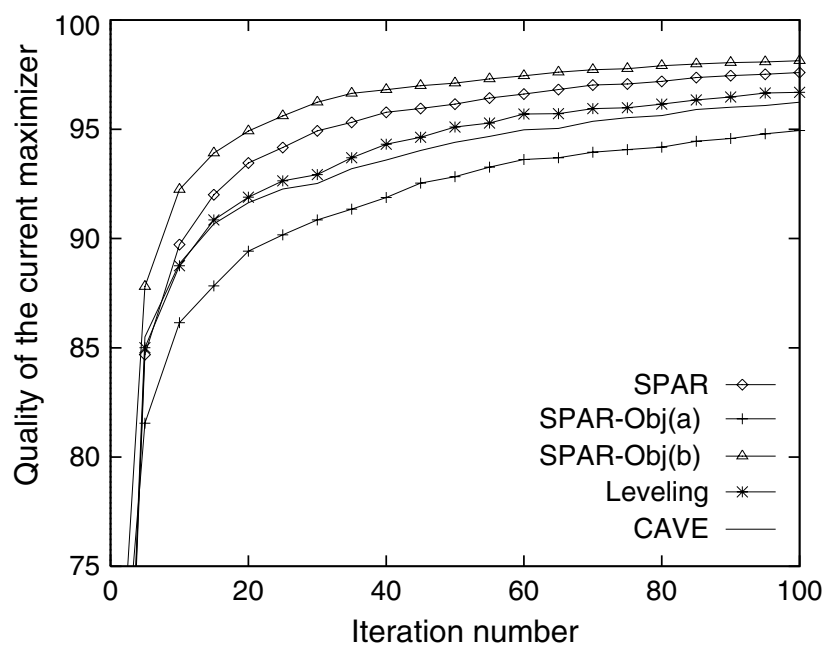

a. Comparison of methods with learning steps.

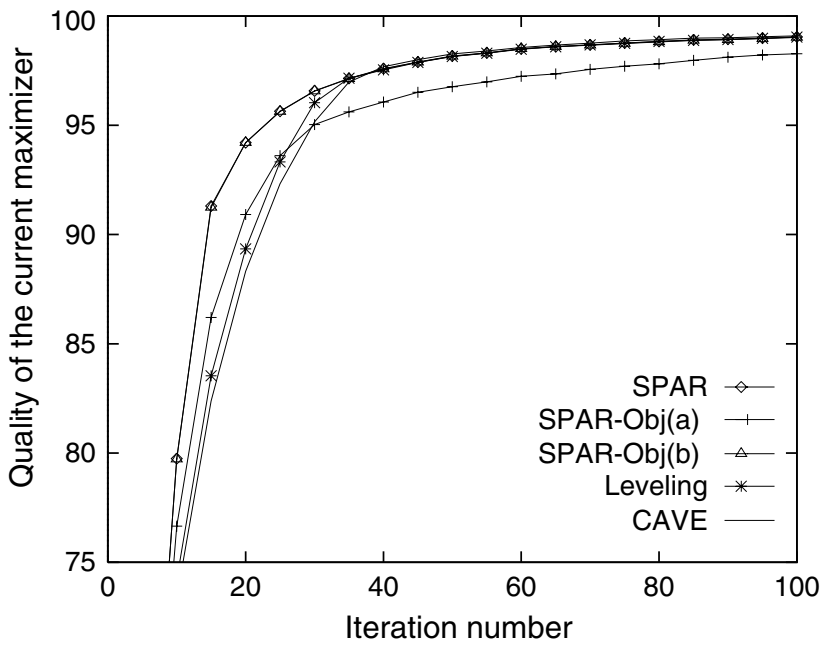

\section{b. Comparison of methods with optimizing steps.}

Figure 1. Comparison of methods with learning (a) and optimizing (b) steps.

This version concentrates its efforts around the maximizers of the approximations, and one might expect that it has a potential of being more efficient. The results are collected in Figure 1(b).

Comparing Figures 1(a) and 1(b), all optimizing methods perform worse than the corresponding learning method at the early iterations. Only after about 30-40 iterations did the versions with optimizing steps take the lead over their learning counterparts. However, the tail performance of the optimizing methods is much better.

Several conclusions can be drawn from our experiments. First, the application of the projection operator facilitates the convergence. It provides an update to a range of values on the basis of the observation obtained at one value. Second, learning is useful especially at earlier iterations. Instead of trying to shoot at the optimal point for the current model, it is better to collect information at randomly selected points from time to time. Third, the indirect use of noisy objective values to correct the subgradients has a positive effect on the convergence, provided that the weight of the additional modification is small. Finally, the learning-based methods provide good approximations to the solutions at early iterations; 
these approximations make them attractive candidates for problems where the cost of one experiment is high.

7. Application to nonseparable resource allocation problems. We now turn our attention to nonseparable problems that arise in the context of two-stage stochastic resource allocation problems. Section 7.1 shows that there exists a separable approximation that will produce an optimal first-stage solution, although there is no guarantee that our algorithm will find this approximation. We also provide a bound on the solution provided by our algorithm. Then, $\$ 7.2$ compares our algorithm, which produces integer solutions, to the solutions produced by several variations of Benders decomposition.

7.1. Outline of the method. Let us start from the following observation. Consider the problem

$$
\max _{x \in X} F(x),
$$

where $X \subset \mathbb{R}^{n}$ is a closed convex set and $F: \mathbb{R}^{n} \rightarrow \mathbb{R}$ is a concave function. Suppose that $\hat{x}$ is the optimal solution of the problem and that $F$ is subdifferentiable at $\hat{x}$. Let us construct a concave separable approximation of $F$ at $\hat{x}$ in the form

$$
\bar{F}^{\hat{x}}(x)=F(\hat{x})+\sum_{i=1}^{n} \bar{f}_{i}^{\hat{x}}\left(x_{i}\right)
$$

where

$$
\bar{f}_{i}^{\hat{x}}\left(x_{i}\right)= \begin{cases}F^{\prime}\left(\hat{x}, e_{i}\right)\left(x_{i}-\hat{x}_{i}\right) & \text { if } x_{i} \geq \hat{x}_{i}, \\ F^{\prime}\left(\hat{x},-e_{i}\right)\left(\hat{x}_{i}-x_{i}\right) & \text { if } x_{i} \leq \hat{x}_{i} .\end{cases}
$$

In the formula above, $F^{\prime}\left(\hat{x}_{i}, d\right)$ denotes the directional derivative of $F$ at $\hat{x}$ in direction $d$, and $e_{i}$ is the $i$ th unit vector in $\mathbb{R}^{n}$. We use $\hat{x}$ as the superscript of $\bar{F}$ to stress that the approximation is constructed at $\hat{x}$.

The point $\hat{x}$ is also the solution of the deterministic approximate problem

$$
\max _{x \in X} \bar{F}^{\hat{x}}(x) .
$$

Indeed, each direction $d$ can be represented as

$$
d=\sum_{i=1}^{n} d_{i} e_{i}=\sum_{i=1}^{n}\left(d_{i}\right)_{+} e_{i}+\sum_{i=1}^{n}\left(d_{i}\right)_{-}\left(-e_{i}\right)
$$

Because the directional derivative is a concave positively homogeneous function, we have

$$
F^{\prime}(\hat{x}, d) \geq \sum_{i=1}^{n}\left(d_{i}\right)_{+} F^{\prime}\left(\hat{x}, e_{i}\right)+\sum_{i=1}^{n}\left(d_{i}\right)_{-} F^{\prime}\left(\hat{x},-e_{i}\right)=\left[\bar{F}^{\hat{x}}\right]^{\prime}(\hat{x}, d) .
$$

By the optimality of $\hat{x}$, the directional derivative $F^{\prime}(\hat{x}, d)$ of $F$ at $\hat{x}$ in any direction $d \in$ cone $(X-\hat{x})$ is nonpositive. Therefore, $\left[\bar{F}^{\hat{x}}\right]^{\prime}(\hat{x}, d) \leq 0$ for every feasible direction $d$, as required.

Consider an arbitrary point $y$ at which such a separable approximation $\bar{F}^{y}(x)$ has been constructed. We have just proved that if $y=\hat{x}$, then $y$ is the solution of (53). The converse statement is not true, in general, and one can easily construct counterexamples in which the separable approximation $\bar{F}^{y}(\cdot)$ constructed at some point $y$ achieves its maximum at $y$, but $F(\cdot)$ does not.

Then, however, we can derive an upper bound on the optimal value of $F(\cdot)$ in $X$ as follows. If $\hat{x}$ is an optimal solution of Problem (52), then

$$
F(\hat{x})-F(y) \leq F^{\prime}(y, \hat{x}-y) \leq-F^{\prime}(y, y-\hat{x}) .
$$


In the second inequality above we have used the concavity of $F^{\prime}(y, \cdot)$. Inequality (54) can be developed at the point $y$ instead of $\hat{x}$ and reads

$$
F^{\prime}(y, d) \geq\left[\bar{F}^{y}\right]^{\prime}(y, d) .
$$

Setting $d=y-\hat{x}$ and combining the last two inequalities, we obtain

$$
F(\hat{x})-F(y) \leq-F^{\prime}(y, y-\hat{x}) \leq-\left[\bar{F}^{y}\right]^{\prime}(y, y-\hat{x}) .
$$

Thus, the following bound on the difference between the optimal value of Problem (52) and the value of $F$ at $y$ holds true:

$$
F(\hat{x})-F(y) \leq-\min _{x \in X}\left[\bar{F}^{y}\right]^{\prime}(y, y-x) .
$$

The quantity at the right-hand side can be easily calculated or estimated, given the current approximate solution $y$ and the piecewise linear separable approximation $\bar{F}^{y}$.

For a general stochastic programming problem with a nonseparable recourse function, our methods do not necessarily converge to the optimal solution. Furthermore, our methods use samples of the directional derivatives in the directions $e_{i}$, rather than exact values, so the error bound will also be an estimate.

For solving $\max _{x \in X} F(x)$, when $F$ is a nonseparable function, our method proceeds as follows: At iteration $k$, our approximation is $\sum_{i=1}^{n} f_{i}^{k}\left(x_{i}\right)$, where each $f_{i}^{k}$ is a one-dimensional, piecewise linear, concave function characterized by the slope vector $v_{i}^{k}=\left(v_{i 1}^{k}, \ldots, v_{i M_{i}}^{k}\right)$ and $M_{i}$ is an upper bound on the variable $x_{i}$. The point $x^{k}$ is the maximizer of $\sum_{i=1}^{n} f_{i}^{k}\left(x_{i}\right)$. We update our approximation using the slopes gathered at $x^{k}$. The procedure is described in Algorithm 3.

Algorithm 3. The optimizing version of SPAR.

Step 0 . Set $v_{i}^{1} \in V$ for all $i=1, \ldots, n, k=1$.

Step 1. Find $x^{k} \in \arg \max _{x \in X} \sum_{i=1}^{n} f_{i}^{k}\left(x_{i}\right)$, where each $f_{i}^{k}$ is defined by the slope vector $v_{i}^{k}$.

Step 2. Observe a random variable $\eta^{k}$ such that

$$
\mathbb{E}\left\{\eta^{k} \mid v^{1}, \ldots, v^{k} ; x^{1}, \ldots, x^{k}\right\} \in \partial F\left(x^{k}\right) \text { a.s. }
$$

Step 3. For each $i=1, \ldots, n$, do the following:

Step 3.1. Calculate the vector $z_{i}^{k} \in \mathbb{R}^{M_{i}}$ as follows:

$$
z_{i s}^{k}= \begin{cases}\left(1-\alpha_{k}\right) v_{i s}^{k}+\alpha_{k} \eta_{i}^{k} & \text { if } s=x_{i}^{k} \\ v_{i s}^{k} & \text { otherwise }\end{cases}
$$

Step 3.2. Calculate $v_{i}^{k+1}=\Pi_{V}\left(z_{i}^{k}\right)$.

Step 4 . Increase $k$ by one and go to Step 1 .

Several remarks are in order. First, as a stopping criterion, one may choose to continue for a specified number of iterations or until $F\left(x^{k}\right)$ does not improve for a certain number of iterations.

Second, we note that there are two sources of error in this approximate procedure. The main source of error is the use of separable approximations, as discussed above. The second source of error is the use of an arbitrary stochastic subgradient $\eta^{k}$ rather than estimates of the forward and backward directional derivatives, as required in $\$ 4$. Nevertheless, the method performs remarkably well on a class of stochastic optimization problems that we discuss below.

Third, many stochastic programming problems lend themselves to compact state variables, and the recourse functions in these problems have considerably fewer dimensions 
than the number of decision variables. For example, in (2), the recourse cost $\mathbb{E} Q(x, \omega)$ depends on $T x$. If the dimension of $T x$ is less than $x$, by writing (2) as

$$
\begin{aligned}
\max & \langle c, x\rangle+\mathbb{E} Q(s, \omega) \\
\text { subject to } & A x=b, \\
& T x-s=0, \\
& x \geq 0
\end{aligned}
$$

where

$$
\begin{aligned}
Q(s, \omega)=\max & \langle q, y\rangle \\
\text { subject to } & W y=h(\omega)-s, \\
& y \geq 0,
\end{aligned}
$$

and building separable approximations of $\mathbb{E} Q(s, \omega)$, we can decrease the number of required approximations.

In this case, the extension of the algorithm we presented above is straightforward. The dimension of $s$ is denoted by $n$. In Step 1 , we set $\left(x^{k}, s^{k}\right) \in \arg \max _{(x, s) \in X}\langle c, x\rangle+$ $\sum_{i=1}^{n} f_{i}^{k}\left(s_{i}\right)$, where $X$ is the feasible set of the first stage problem. In Step 2, $\eta^{k}$ has to satisfy $\mathbb{E}\left\{\eta^{k} \mid v^{1}, \ldots, v^{k} ; s^{1}, \ldots, s^{k}\right\} \in \partial \mathbb{E}\left\{Q\left(s^{k}, \omega\right)\right\}$. One can choose $\eta^{k}$ to be the Lagrange multipliers associated with the constraints of the second-stage problem for a certain realization of $\omega$, say $\omega^{k}$ and for a certain value of $s^{k}$. If $\omega^{k}$ is independent of all previously used realizations of $\omega$, then $\mathbb{E}\left\{\eta^{k} \mid v^{1}, \ldots, v^{k} ; s^{1}, \ldots, s^{k}\right\} \in \partial \mathbb{E}\left\{Q\left(s^{k}, \omega\right)\right\}$ is easily satisfied.

7.2. Numerical illustration. We illustrate our method using the following two-stage stochastic programming problem:

$$
\begin{aligned}
\max & \sum_{i \in \mathcal{F}} \sum_{j \in \mathcal{I} \cup \mathscr{C}} c_{i j} x_{i j}+\mathbb{E} Q(s, D) \\
\text { subject to } & \sum_{j \in \mathcal{I} \cup \mathscr{C}} x_{i j} \leq p_{i}, \quad i \in \mathscr{F}, \\
& \sum_{i \in \mathcal{F}} x_{i j}-s_{j}=0, \quad j \in \mathscr{F} \cup \mathscr{C}, \\
& x_{i j}, s_{j} \geq 0,
\end{aligned}
$$

where $Q(s, D)$ is the optimal value of the second-stage problem:

$$
\begin{aligned}
\max & \sum_{i \in \mathscr{I} \cup \mathscr{C}} \sum_{j \in \mathscr{C}} d_{i j} y_{i j}+\sum_{i \in \mathscr{C}} \sum_{l \in \mathscr{L}} r_{i}^{l} z_{i}^{l} \\
\text { subject to } & \sum_{j \in \mathscr{C}} y_{i j} \leq s_{i}, \quad i \in \mathscr{I} \cup \mathscr{C}, \\
& \sum_{i \in \mathscr{I} \cup \mathscr{C}} y_{i j}-\sum_{l \in \mathscr{L}} z_{j}^{l} \geq 0, \quad j \in \mathscr{C}, \\
& z_{j}^{l} \leq D_{j}^{l}, \quad l \in \mathscr{L}, \quad j \in \mathscr{C}, \\
& y_{i j}, z_{j}^{l} \geq 0 .
\end{aligned}
$$

The problem above can be interpreted as follows: There is a set of production facilities (with warehouses) $\mathscr{I}$ and a set of customers $\mathscr{C}$. At the first stage, an amount $x_{i j}$ is transported from production facility $i$ to a warehouse or customer location $j$, before the demand realizations at the customer locations become known. After the realizations of the demand 
at the customer locations are observed, we move an amount $y_{i j}$ from location $i$ to customer location $j$. At each customer location we face different types of demands, indexed by $l \in \mathscr{L}$ : $D_{i}^{l}$ is the demand of type $l$ at location $j$. We serve $z_{j}^{l}$ units of demand of type $l$ at location $j$; the excess demand, if any, is lost. The production capacity of facility $i$ is denoted by $p_{i}$.

For the first-stage costs, we set $c_{i j}=c_{0}+c_{1} \delta_{i j}$, where $\delta_{i j}$ is the Euclidean distance between locations $i$ and $j$, and $c_{0}$ can be interpreted as the unit production cost and $c_{1}$ is the transportation cost applied on a per mile basis. For the second-stage costs, we have

$$
d_{i j}= \begin{cases}d_{1} \delta_{i j} & \text { if } i \in \mathscr{F} \text { or } i=j, \\ d_{0}+d_{1} \delta_{i j} & \text { if } i \in \mathscr{C} \text { and } i \neq j .\end{cases}
$$

$d_{0}$ represents the fixed charge for shipping a unit of the product from one customer location to another customer location, and $d_{1}$ is the per mile cost of transportation in the second stage. For every demand type $l$ occurring in location $i$, we associate a revenue $r_{i}^{l}$. Our test problems differ in cost parameters and $|\mathscr{J} \cup \mathscr{C}|$, which determines the dimensionality of the recourse function. Each test problem has 100 possible demand scenarios.

As a benchmark, we use three well-known Benders decomposition-based stochastic programming algorithms: L-shaped decomposition (LSD) (Van Slyke and Wets 1969), stochastic decomposition (SD) (Higle and Sen 1991), and cutting plane partial sampling (CUPPS) (Chen and Powell 1999).

Our focus is on the rate of convergence, measured by the improvement in the objective function as the number of iterations or the CPU time increases. To measure the rate of convergence of different methods, we ran each algorithm for 25, 100, 500, 1,000, and 5,000 iterations. For our separable approximations, the number of iterations refer to the number of demand samples used. For LSD, SD, and CUPPS, the number of iterations refer to the number of cuts used to approximate the recourse function. Having constructed a recourse approximation at iteration $k$, say $\hat{Q}^{k}$, we find $\left(x^{k}, s^{k}\right) \in \arg \max \sum_{i, j \in \mathcal{J}} c_{i j} x_{i j}+\hat{Q}^{k}(s)$. Then, we compute $\sum_{i, j \in \mathcal{J}} c_{i j} x_{i j}^{k}+\mathbb{E} Q\left(s^{k}, \omega\right)$ to measure the performance of the solution $\left(x^{k}, s^{k}\right)$ provided by the approximation $\hat{Q}^{k}$.

The results are summarized in Table 1. The numbers in the table represent the percent deviation between the optimal objective value and the objective value corresponding to the solution obtained after a certain number of iterations. For all problem instances, we use LSD to find the optimal solution. Table 1 also gives the CPU time per iteration. We present results on 10 problems. Six of these problems vary in cost parameters, and four vary in the dimensionality of the recourse function.

The results indicate that for the problem class we consider, SPAR is able to produce high-quality solutions rather quickly, and provides consistent performance over different sets of problem parameters. In particular, the consistent performance of SPAR over problems with different numbers of locations may make it appealing for large-scale applications. Nevertheless, our numerical results are limited to a specific problem class and one should be cautious about extending our findings to other problem classes. However, as a result of Equation (55), SPAR provides an estimate of the optimality bound at every point it generates.

In view of these results, SPAR is a promising approach for the allocation of indivisible resources under uncertainty, but more comprehensive numerical work is needed before using it in a particular problem context. Finally, we note that due to its simplicity and fast runtimes, SPAR can be used as an initialization routine for stochastic programming approaches that can exploit high-quality feasible solutions. For example, the recourse approximation provided by SPAR can be used to initialize that of SD or LSD, and CUPPS can start by constructing a support of the recourse function at the solution provided by SPAR. 
Powell, Ruszczyński, and Topaloglu: Learning Algorithms for Separable Approximations Mathematics of Operations Research 29(4), pp. 814-836, (C) 2004 INFORMS

TABlE 1. Percent error over optimal with different first-stage costs.

\begin{tabular}{|c|c|c|c|c|c|c|c|}
\hline \multirow[b]{2}{*}{ Problem } & \multirow[b]{2}{*}{ Method } & \multicolumn{5}{|c|}{ Number of iterations } & \multirow[b]{2}{*}{ Sec./iter } \\
\hline & & 25 & 100 & 500 & 1,000 & 5,000 & \\
\hline $\begin{array}{l}\text { Cost. } \\
\text { param. I }\end{array}$ & $\begin{array}{l}\text { SPAR } \\
\text { LSD } \\
\text { CUPPS } \\
\text { SD }\end{array}$ & $\begin{array}{l}10.18 \\
29.88 \\
20.74 \\
47.63\end{array}$ & $\begin{array}{r}0.81 \\
11.68 \\
9.86 \\
15.64\end{array}$ & $\begin{array}{r}0.21 \\
0.44 \\
5.21 \\
11.42\end{array}$ & $\begin{array}{l}0.15 \\
0.03 \\
2.39 \\
7.56\end{array}$ & $\begin{array}{l}0 \\
2.48\end{array}$ & $\begin{array}{l}0.07 \\
2.41 \\
0.37 \\
0.46\end{array}$ \\
\hline $\begin{array}{l}\text { Cost. } \\
\text { param. II }\end{array}$ & $\begin{array}{l}\text { SPAR } \\
\text { LSD } \\
\text { CUPPS } \\
\text { SD }\end{array}$ & $\begin{array}{r}9.28 \\
44.19 \\
49.43 \\
24.76\end{array}$ & $\begin{array}{r}0.77 \\
8.28 \\
13.58 \\
17.8\end{array}$ & $\begin{array}{l}0.23 \\
0.49 \\
6.17 \\
8.56\end{array}$ & $\begin{array}{l}0.26 \\
0.05 \\
1.95 \\
8.54\end{array}$ & $\begin{array}{l}0 \\
1.62\end{array}$ & $\begin{array}{l}0.06 \\
2.46 \\
0.37 \\
0.46\end{array}$ \\
\hline $\begin{array}{l}\text { Cost. } \\
\text { param. III }\end{array}$ & $\begin{array}{l}\text { SPAR } \\
\text { LSD } \\
\text { CUPPS } \\
\text { SD }\end{array}$ & $\begin{array}{l}10.72 \\
37.07 \\
36.36 \\
35.37\end{array}$ & $\begin{array}{r}1.64 \\
8.16 \\
10.99 \\
17.52\end{array}$ & $\begin{array}{l}0.39 \\
0.5 \\
6.3 \\
9.14\end{array}$ & $\begin{array}{l}0.44 \\
0 \\
2.25 \\
6.53\end{array}$ & $\begin{array}{l}0.1 \\
0 \\
2.28\end{array}$ & $\begin{array}{l}0.05 \\
2.51 \\
0.37 \\
0.45\end{array}$ \\
\hline $\begin{array}{l}\text { Cost. } \\
\text { param. IV }\end{array}$ & $\begin{array}{l}\text { SPAR } \\
\text { LSD } \\
\text { CUPPS } \\
\text { SD }\end{array}$ & $\begin{array}{l}10.43 \\
36.18 \\
41.79 \\
57.06\end{array}$ & $\begin{array}{r}2.41 \\
6.72 \\
22.02 \\
25.4\end{array}$ & $\begin{array}{r}0.67 \\
0.46 \\
9.14 \\
23.11\end{array}$ & $\begin{array}{l}0.65 \\
0 \\
2.94 \\
12.02\end{array}$ & $\begin{array}{l}0.08 \\
0 \\
4.45\end{array}$ & $\begin{array}{l}0.04 \\
2.03 \\
0.64 \\
0.45\end{array}$ \\
\hline $\begin{array}{l}\text { Cost. } \\
\text { param. V }\end{array}$ & $\begin{array}{l}\text { SPAR } \\
\text { LSD } \\
\text { CUPPS } \\
\text { SD }\end{array}$ & $\begin{array}{r}9.58 \\
29.53 \\
36.28 \\
25.37\end{array}$ & $\begin{array}{l}3.61 \\
11.49 \\
21.34 \\
22\end{array}$ & $\begin{array}{r}0.53 \\
0.33 \\
9.26 \\
23.89\end{array}$ & $\begin{array}{c}0.74 \\
0 \\
2.5 \\
25.93\end{array}$ & $\begin{array}{l}0.07 \\
0 \\
3.25\end{array}$ & $\begin{array}{l}0.04 \\
1.68 \\
0.64 \\
0.44\end{array}$ \\
\hline $\begin{array}{l}\text { Cost. } \\
\text { param. VI }\end{array}$ & $\begin{array}{l}\text { SPAR } \\
\text { LSD } \\
\text { CUPPS } \\
\text { SD }\end{array}$ & $\begin{array}{r}8.95 \\
40.68 \\
38.97 \\
40.64\end{array}$ & $\begin{array}{r}4.42 \\
1.91 \\
6.02 \\
11.37\end{array}$ & $\begin{array}{l}0.75 \\
0 \\
4.77 \\
9.22\end{array}$ & $\begin{array}{l}0.87 \\
0.53 \\
6.78\end{array}$ & 0.09 & $\begin{array}{l}0.04 \\
0.93 \\
0.64 \\
0.43\end{array}$ \\
\hline$|\mathscr{I} \cup \mathscr{C}|=10$ & $\begin{array}{l}\text { SPAR } \\
\text { LSD } \\
\text { CUPPS } \\
\text { SD }\end{array}$ & $\begin{array}{c}18.65 \\
3.3 \\
5.84 \\
45.45\end{array}$ & $\begin{array}{l}7.07 \\
0 \\
0.5 \\
11.35\end{array}$ & $\begin{array}{l}0.48 \\
0 \\
2.3\end{array}$ & 0.28 & 0.15 & $\begin{array}{l}0.00 \\
0.06 \\
0.07 \\
0.10\end{array}$ \\
\hline$|\mathscr{I} \cup \mathscr{C}|=25$ & $\begin{array}{l}\text { SPAR } \\
\text { LSD } \\
\text { CUPPS } \\
\text { SD }\end{array}$ & $\begin{array}{r}11.73 \\
19.88 \\
8.27 \\
40.55\end{array}$ & $\begin{array}{r}2.92 \\
2.14 \\
4.33 \\
22.22\end{array}$ & $\begin{array}{l}0.34 \\
0 \\
1.47 \\
4.24\end{array}$ & $\begin{array}{l}0.16 \\
4.8\end{array}$ & 0.06 & $\begin{array}{l}0.02 \\
0.26 \\
0.31 \\
0.22\end{array}$ \\
\hline$|\mathscr{I} \cup \mathscr{C}|=50$ & $\begin{array}{l}\text { SPAR } \\
\text { LSD } \\
\text { CUPPS } \\
\text { SD }\end{array}$ & $\begin{array}{r}9.99 \\
42.56 \\
34.93 \\
43.18\end{array}$ & $\begin{array}{c}1.18 \\
6.07 \\
19.3 \\
17.94\end{array}$ & $\begin{array}{l}0.26 \\
0.52 \\
5.09 \\
5.91\end{array}$ & $\begin{array}{l}0.3 \\
0.04 \\
1.38 \\
6.25\end{array}$ & $\begin{array}{l}0 \\
1.02\end{array}$ & $\begin{array}{l}0.06 \\
2.51 \\
0.37 \\
0.46\end{array}$ \\
\hline$|\mathscr{I} \cup \mathscr{C}|=100^{*}$ & $\begin{array}{l}\text { SPAR } \\
\text { LSD } \\
\text { CUPPS } \\
\text { SD }\end{array}$ & $\begin{array}{r}8.74 \\
74.52 \\
54.59 \\
62.63\end{array}$ & $\begin{array}{c}1.2 \\
26.21 \\
23.99 \\
40.73\end{array}$ & $\begin{array}{r}0.16 \\
2.32 \\
14.68 \\
15.22\end{array}$ & $\begin{array}{r}0.05 \\
0.85 \\
14.13 \\
17.43\end{array}$ & $\begin{array}{l}0 \\
0.02 \\
0.91 \\
9.42\end{array}$ & $\begin{array}{r}0.22 \\
10.15 \\
1.42 \\
1.30\end{array}$ \\
\hline
\end{tabular}

Note. Figures represent the deviation from the best objective value known.

${ }^{*}$ Optimal solution not found.

Acknowledgments The authors thank the reviewers and editors for comments that improved the presentation of the paper. The first author was supported in part by Grant AFOSR-F49620-93-1-0098 from the Air Force Office of Scientific Research. The second author was supported by the National Science Foundation under Grant DMS-0303545. The third author was supported by the National Science Foundation under Grant DMI-0422133.

\section{References}

Birge, J., S. W. Wallace. 1988. A separable piecewise linear upper bound for stochastic linear programs. SIAM J. Control Optim. 26(3) 1-14.

Chen, Z.-L., W. Powell. 1999. A convergent cutting-plane and partial-sampling algorithm for multistage linear programs with recourse. J. Optim. Theory Appl. 103(3) 497-524. 
Cheung, R., W. B. Powell. 1996. An algorithm for multistage dynamic networks with random arc capacities, with an application to dynamic fleet management. Oper. Res. 44(6) 951-963.

Cheung, R. K.-M., W. B. Powell. 2000. SHAPE: A stochastic hybrid approximation procedure for two-stage stochastic programs. Oper. Res. 48(1) 73-79.

Culioli, J.-C., G. Cohen. 1990. Decomposition/coordination algorithms in stochastic optimization. SIAM J. Control Optim. 28 1372-1403.

Ding, X., M. L. Puterman, A. Bisi. 2002. The censored newsvendor and the optimal acquisition of information. Oper. Res. 50(3) 517-527.

Doob, J. L. 1953. Stochastic Processes. John Wiley and Sons, New York.

Ermoliev, Y. 1988. Stochastic quasigradient methods. Y. Ermoliev, R. Wets, eds. Numerical Techniques for Stochastic Optimization. Springer-Verlag, Berlin, Germany.

Frantzeskakis, L., W. B. Powell. 1990. A successive linear approximation procedure for stochastic dynamic vehicle allocation problems. Transportation Sci. 24(1) 40-57.

Godfrey, G. A., W. B. Powell. 2001. An adaptive, distribution-free approximation for the newsvendor problem with censored demands, with applications to inventory and distribution problems. Management Sci. 47(8) $1101-1112$

Higle, J., S. Sen. 1991. Stochastic decomposition: An algorithm for two stage linear programs with recourse. Math. Oper. Res. 16(3) 650-669.

Kleywegt, A. J., A. Shapiro, T. Homem-De-Mello. 2002. The sample average approximation method for stochastic discrete optimization. SIAM J. Optim. 12 479-502.

Korf, L. A., R. J. B. Wets. 2001. Random LSC functions: An ergodic theorem. Math. Oper. Res. 26(2) 421-445.

Kushner, H. J., G. G. Yin. 1997. Stochastic Approximation Algorithms and Applications. Springer-Verlag, New York.

Laporte, G., F. Louveaux. 1993. The integer L-shaped method for stochastic integer programs with complete recourse. Oper. Res. Lett. 13(3) 133-142.

Porteus, E. L. 1990. Stochastic inventory theory. Handbooks in Operations Research and Management Science: Stochastic Models, Vol. 2. North-Holland, Amsterdam, The Netherlands, 605-652.

Powell, W. B. 1986. A stochastic model of the dynamic vehicle allocation problem. Transportation Sci. 20 117-129.

Powell, W. B. 1987. An operational planning model for the dynamic vehicle allocation problem with uncertain demands. Transportation Res. 21B 217-232.

Powell, W. B. 1988. A comparative review of alternative algorithms for the dynamic vehicle allocation problem. B. Golden, A. Assad, eds. Vehicle Routing: Methods and Studies. North-Holland, Amsterdam, The Netherlands, 249-292.

Powell, W. B., T. A. Carvalho. 1998. Dynamic control of logistics queueing networks for large-scale fleet management. Transportation Sci. 32(2) 90-109.

Powell, W. B., R. Cheung. 1994. Stochastic programs over trees with random arc capacities. Networks 24 161-175.

Powell, W. B., H. Topaloglu. 2003. Stochastic programming in transportation and logistics. A. Ruszczyński, A. Shapiro, eds. Handbooks in Operations Research and Management Science: Stochastic Programming. Elsevier, Amsterdam, The Netherlands, 555-635.

Righter, R. 1989. A resource allocation problem in a random environment. Oper. Res. 37 329-338.

Ruszczyński, A. 1980. Feasible direction methods for stochastic programming problems. Math. Programming 19 220-229.

Ruszczyński, A. 1986. A method of feasible directions for solving nonsmooth stochastic programming problems. F. Archetti, G. DiPillo, M. Lucertini, eds. Stochastic Programming. Springer-Verlag, Berlin, Germany, 258-271.

Ruszczyński, A. 1987. A linearization method for nonsmooth stochastic programming problems. Math. Oper. Res. 12(1) 32-49.

Ruszczyński, A. 2003. Decomposition methods. A. Ruszczyński, A. Shapiro, eds. Handbooks in Operations Research and Management Science: Stochastic Programming. Elsevier, Amsterdam, The Netherlands.

Shapiro, A., T. Homem-De-Mello. 2000. On the rate of convergence of optimal solutions of Monte Carlo approximations of stochastic programs. SIAM J. Optim. $1170-86$.

Topaloglu, H., W. B. Powell. 2003. An algorithm for approximating piecewise linear concave functions from sample gradients. Oper. Res. Lett. 31(1) 66-76.

Van Slyke, R., R. Wets. 1969. L-shaped linear programs with applications to optimal control and stochastic programming. SIAM J. Appl. Math. 17(4) 638-663.

Wallace, S. 1986. Solving stochastic programs with network recourse. Networks 16 295-317.

Wallace, S. 1987. A piecewise linear upper bound on the network recourse function. Math. Programming 38 133-146. 